\title{
COMUNICAÇÃO DA EQUIPE INTERDISCIPLINAR NA ASSISTÊNCIA AOS PACIENTES CIRÚRGICOS
}

\author{
COMUNICACIÓN DEL EQUIPO INTERDISCIPLINAR EN LA \\ ASISTENCIA A LOS PACIENTES QUIRÚRGICOS
}

\author{
COMMUNICATION OF THE INTERDISCIPLINARY TEAM IN ASSISTANCE TO \\ SURGICAL PATIENTS
}

Helena Bazanelli PREBIANCHI ${ }^{1}$

RESUMO: O prontuário do paciente é uma das formas de comunicação entre os membros da equipe interdisciplinar. Objetivou-se analisar os prontuários de pacientes submetidos à cirurgias eletivas, no período de outubro de 2014 a fevereiro de 2015. Foi realizada uma pesquisa documental incluindo 29 prontuários. Os resultados mostraram que todos os prontuários eram em preenchidos manualmente; 22 continham a assinatura do médico responsável; 27 continham o Termo de Consentimento; o registro de Evolução Multidisciplinar foi realizado em 18 prontuários, englobando diferentes áreas; as notas psicológicas foram redigidas de modo claro, mas faltavam informações sobre a subjetividade. Discutiu-se que se o registro no prontuário é escasso e inadequado à segurança do paciente, da instituição e da equipe ficam comprometidas e há dificuldade para mensurar os resultados assistenciais. Concluímos que os profissionais da equipe mantêm o caráter de especialistas, com forte delimitação do seu papel individual graças à distorção da lógica da interdisciplinaridade.

PALAVRAS-CHAVE: Paciente cirúrgico. Equipe interdisciplinar. Prontuário. Psicólogo. Hospitalar.

RESUMEN: El prontuario del paciente es una de las formas de comunicación entre los miembros del equipo interdisciplinario. Se objetivó analizar los prontuarios de pacientes sometidos a las cirugías electivas, en el período de octubre de 2014 a febrero de 2015. Se realizó una investigación documental incluyendo 29 prontuarios. Los resultados mostraron que todos los prontuarios se llenaban manualmente; 22 contenían la firma del médico responsable; 27 contenían el Término de Consentimiento; el registro de Evolución Multidisciplinar fue realizado en 18 registros, englobando diferentes áreas; las notas psicológicas fueron redactadas de modo claro, pero faltaban informaciones sobre la subjetividad. Se discutió que si el registro en el prontuario es escaso e inadecuado a la seguridad del paciente, de la institución y del equipo quedan comprometidas y hay dificultad para medir los resultados asistenciales. Se concluyó que los profesionales del equipo mantienen el carácter de especialistas, con fuerte

${ }^{1}$ Pontifícia Universidade Católica de Campinas (PUC-Campinas), Campinas - SP - Brasil. Docente do Programa de Pós-Graduação em Psicologia e professora titular da Faculdade de Psicologia. E-mail: helenabp@puc-campinas.edu.br 
delimitación de su papel individual gracias a la distorsión de la lógica de la interdisciplinariedad.

PALABRAS-CLAVE: Paciente quirúrgico. Equipo interdisciplinario. Pronóstico. Psicológo. Hospitalario.

ABSTRACT: The patient records is one of the main forms of communication between members of the interdisciplinary team. Objective: to analyze the medical records of patients undergoing elective surgeries, from October 2014 to February 2015. Method: a documentary research including 29 medical records. Results: all medical records were filled in manually; 27 of the medical records contained the consent form, the Multidisciplinary Evolution registration was carried out in 18 and encompassed different areas. Conclusion: It was concluded that the hospital, context of the research, the team members maintain the character of experts, with strong delineation of their individual role thanks to the distortion of the interdisciplinary logic. It is recommended to be given training by the institution to the team members, in order to enlighten them and make them aware of the issue and improvement in the forms and hospital management mechanisms in order to ensure comprehensive care to patients.

KEYWORDS: Surgical patient. Interdisciplinary team. Patient Record. Hospital psychologist.

\section{Introdução}

Toda e qualquer intervenção cirúrgica é uma situação crítica que expõe o indivíduo a um estresse físico e emocional (Juan, 2005). Diante da necessidade da realização de uma intervenção cirúrgica, o paciente sente sua integridade física e psicológica ameaçadas, manifestando sentimentos de impotência, medo da morte e de possíveis mudanças que podem decorrer do processo cirúrgico (BROERING; CREPALDI, 2011).

Quando os aspectos psicológicos não são considerados na situação de tratamento cirúrgico, poderá haver aumento da predisposição para complicações emocionais que prejudicam a convalescença, chegando a intensificar, em algumas situações, a morbidade no período pós-operatório (FERRACINI; PREBIANCHI, 2016).

Relatos de pacientes expostos a procedimentos cirúrgicos apontam que os principais fatores desencadeantes de ansiedade incluem: a) percepção antecipada de dor e desconforto; b) espera passiva pelo início do procedimento; c) separação da família e sentimentos de abandono; d) possível perda, mesmo que temporária, de autonomia; e) medo da morte, de sequelas, do procedimento de anestesia e do risco de alta prematura; e f) o procedimento cirúrgico como um todo (TURRA et al, 2011). 
O psicólogo hospitalar trabalha nesse contexto, procurando amenizar sentimentos que possam interferir na recuperação do paciente, assim como o prepara para situações que provavelmente irá se deparar ao longo de sua internação, com o objetivo de proporcionar confiança e tranqüilidade.

Uma visão completa da complexidade do ato cirúrgico envolve cinco tipos de variáveis interdependentes: as físico-químicas, as biológicas, as psicológicas, as sociais e as culturais; cada qual com suas características particulares. Assim, o ato cirúrgico, envolve desde as reações da pessoa diante do diagnóstico, da necessidade da cirurgia, associadas ao funcionamento e à organização do ambiente e da equipe, até o desenvolvimento da cirurgia, as técnicas relacionadas, seus resultados e a cultura em que o paciente está inserido.

Sendo um evento multideterminado, com diversas variáveis combinadas que interagem constantemente entre si, a situação cirúrgica requer a interação efetiva entre a equipe multiprofissional e interdisciplinar envolvida na prestação da assistência, na busca pelas reais necessidades desses pacientes. Nesse sentido, é preciso desenvolver, estabelecer e aperfeiçoar modos sistematizados de atendimento à saúde do paciente cirúrgico, que estruturem e melhorem o sistema de comunicação interdisciplinar, de modo a tornar a assistência menos fragmentada e mais integral (TURRA et al, 2011).

O prontuário do paciente é uma das principais formas de comunicação entre os membros da equipe interdisciplinar e, se devidamente preenchido, há uma garantia da qualidade da assistência e da facilidade de recuperação de informações sobre o paciente, quando necessário (MESQUITA; DESLANDES, 2010; KLUCK; GUIMARÃES, 2014).

$\mathrm{Na}$ maior parte dos hospitais do país, o prontuário é ainda chamado de prontuário médico. Klück e Guimarães (2014), afirmam que, considerando-se que, em nível internacional, essa abordagem está se modificando para uma visão que parte do usuário (provedor de todas as informações constantes no prontuário e principal beneficiário dessas), o adjetivo 'médico' não parece refletir adequadamente a situação e, por esse motivo, está sendo progressivamente chamado de "prontuário do paciente".

No Brasil, o Hospital da Universidade de São Paulo foi o primeiro hospital a implantar o serviço de arquivo médico, em 1943, seguido pela Santa Casa de Santos, em 1945, e pelo Hospital dos Servidores do Estado do Rio de Janeiro. A partir da Lei Alípio Correia Netto, de 1952, os hospitais públicos e filantrópicos passaram a ser obrigados a arquivar as histórias clínicas de forma adequada, como condição para 
recebimento das subvenções públicas. Nos dias de hoje, o Programa Brasileiro de Acreditação Hospitalar exige a existência de prontuários completos, com informações de qualidade e disponíveis nas áreas assistenciais para certificação dos hospitais.

O Conselho Federal de Medicina (2002, s/p), define prontuário como sendo,

[...] o documento único constituído de um conjunto de informações, sinais e imagens registradas, geradas a partir de fatos, acontecimentos e situações sobre a saúde do paciente e a assistência a ele prestada, de caráter legal, sigiloso e cientifico, que possibilita a comunicação entre os membros da equipe multiprofissional e a continuidade prestada ao indivíduo.

Segundo Dall'aglio e Martins (2011), quer seja no suporte papel (preenchido manualmente) ou em prontuários eletrônicos, a qualidade das informações prestadas é fundamental, já que, de acordo com o Conselho Federal de Medicina, esse prontuário é de propriedade do paciente e toda documentação referente ao atendimento prestado ao assistido, pertence a este, servindo a ele, à assistência e à pesquisa. A precisão das informações é direito do paciente e dever daqueles que incluem informações no prontuário.

Sampaio e Silva (2010) acreditam que o prontuário mais do que um elemento de comunicação entre os setores internos e entre a instituição e os pacientes, é um instrumento capaz de mensurar diretamente o tipo e qualidade da relação entre os profissionais e os pacientes.

Além de ser um meio de comunicação entre os diferentes profissionais da equipe e em um recurso indispensável para assegurar a continuidade do atendimento, tanto durante uma internação como no período entre as consultas de ambulatório, o prontuário possibilita a realização de pesquisas nas várias áreas da saúde.

Mesquita e Deslandes (2010) apontam que o prontuário é um instrumento jurídico de registro de propriedade do paciente, atuando como mediador da comunicação intra-equipe de saúde e da comunicação dessa equipe com o usuário. Para Klück e Guimarães (2014), o prontuário é também um suporte para a área administrativa do hospital, uma vez que seus registros são documentos essenciais para a elucidação de questões jurídicas e éticas cada vez mais frequentes, envolvendo pacientes, serviços de saúde e seus profissionais.

No que se refere ao trabalho do psicólogo nas instituições de saúde, de acordo com Calvetti, Fighera e Muller (2008), o registro no prontuário do paciente suscita 
reflexões quanto aos aspectos éticos presentes nas circunstâncias cotidianas da prática profissional, envolvendo, nesse caso, principalmente, as questões sobre o sigilo e a privacidade. As intervenções interdisciplinares não devem prescindir da ética e da responsabilidade do psicólogo de auxiliar na adesão a tratamentos e redução do impacto da doença sobre o funcionamento do indivíduo (GIOIA-MARTINS; ROCHA, 2001).

Numa proposta de modelo de anotações psicológicas em prontuário, Almeida, Anderson e Costa Junior (2008), indicam que as informações compartilhadas pelo psicólogo devem ser redigidas de modo claro e acurado, serem pertinentes e acessíveis a toda a equipe de saúde, respeitando a confidencialidade requerida do profissional de Psicologia, o qual deve considerar o que é importante relatar no prontuário do paciente e o que é necessário relatar apenas em notas de evolução psicológica restritas à utilização do serviço de Psicologia.

Nascimento et al. (2006) propõem um modelo de anotação psiquiátrica em prontuário, destacando o cuidado que deve ser tomado com as normas de sigilo profissional de cada categoria bem como com a utilização de linguagem adequada ao entendimento dos outros profissionais que compõem a equipe.

Em estudo de Azevedo e Thomas (2002), algumas diretrizes para a construção do registro psicológico em prontuário, expõem a necessidade de se formular um modelo de anotações de modo a facilitar o entendimento do quadro do paciente.

Especificamente, em relação à situação cirúrgica, a atuação do psicólogo pressupõe o desenvolvimento de formas de atendimento sistematizadas, integradas e flexíveis ao contexto institucional em que está o paciente cirúrgico, esclarecendo o papel das variáveis psicológicas na adesão às terapêuticas e no enfrentamento dos procedimentos cirúrgicos, com potencial de impacto positivo sobre a qualidade total da assistência.

A integração com os demais profissionais da equipe só pode ser assegurada através de mecanismos e estratégias eficientes de comunicação. Considerando-se que o prontuário do paciente é uma das principais formas de comunicação entre os membros da equipe interdisciplinar, seu estudo pode proporcionar uma compreensão sobre a comunicação dessa equipe e a participação do psicólogo na mesma.

Este trabalho objetivou investigar a comunicação entre os membros da equipe interdisciplinar na assistência aos pacientes adultos submetidos a cirurgias eletivas, através dos registros nos prontuários a partir da internação desses pacientes. 


\section{Método}

\section{Contexto}

A pesquisa documental e retrospectiva, abrangendo as internações na Clínica Cirúrgica em setembro de 2014 à Janeiro de 2015, foi realizada em um hospital universitário de uma cidade do interior do Estado de São Paulo. O mesmo conta com 353 leitos ativos, sendo 243 destinados exclusivamente ao convênio do Sistema Único de Saúde (SUS). Participa no atendimento à população com uma média anual de 20 mil consultas ambulatoriais, 15 mil atendimentos nas unidades de urgência e emergência, 1250 procedimentos cirúrgicos, além de 1600 internações.

Em relação ao Serviço de Psicologia, o Hospital contava, à época do estudo, com uma equipe de três psicólogos assistenciais contratados e quatro psicólogos do Programa de Residência em Psicologia da Saúde/Hospitalar.

\section{Instrumentos}

As informações foram obtidas por meio dos registros nos prontuários, os quais são compostos por: Ficha de internação, Termo de Consentimento, Prescrição Médica, Evolução Médica e Evolução Multidisciplinar.

\section{Procedimento}

O presente estudo é parte de uma pesquisa maior, desenvolvida pela autora sobre atenção psicológica aos pacientes cirúrgicos adultos e infantis em um hospital- escola, a qual foi desenvolvida de acordo maior com as normas preconizadas pela resolução 196/96 do Conselho Nacional de Saúde, que trata de pesquisas envolvendo seres humanos. Desse modo, a pesquisa foi aprovada pelo Comitê de Ética [nome da instituição omitido para evitar identificação] (N. CEP 410.662).

Após ciência do responsável pelo Serviço de Psicologia do hospital, sobre a utilização dos documentos abarcados pela pesquisa, mensalmente, eram identificados por uma das psicólogas assistenciais, aqueles prontuários referentes a pacientes 
cirúrgicos os quais então, eram disponibilizados à pesquisadora que os examinava em uma sala, nas dependências do hospital.

Durante o período do estudo, 47 prontuários foram identificados para inclusão na pesquisa, contudo apenas 29 puderam ser, de fato, incluídos, pois 18 deles não foram encontrados pelo setor responsável.

\section{Análise dos dados}

Os prontuários foram analisados em termos de tipo (suporte papel ou eletrônico), completude de sua composição e dos registros feitos pelos profissionais da equipe.

Os registros dos profissionais foram analisados em termos de frequência de sua ocorrência, linguagem utilizada e conteúdo (no caso dos profissionais de psicologia).

\section{Resultados e discussão}

A primeira constatação no material analisado refere-se ao tipo de prontuário utilizado: todos os prontuários abarcados no estudo foram do tipo tradicional ou suporte papel. Este resultado é congruente com os achados de Silva e Tavares-Neto (2007), num estudo que avaliou os prontuários médicos de hospitais de ensino no Brasil e concluiu que o tipo predominante ainda é em suporte de papel, nenhum em suporte eletrônico. Para os autores, esses achados revelam que para além das dificuldades financeiras, os hospitais de ensino brasileiros, sofrem as conseqüências de políticas públicas que não favorecem o aprimoramento da gestão hospitalar.

Similarmente, Dall'aglio e Martins (2011), em seu trabalho de avaliação de dados de prontuários do módulo urgência e emergência do hospital de clínicas da Universidade Federal de Uberlândia, encontraram que os mesmos eram somente em suporte papel, preenchidos manualmente. Ainda que neste tipo de prontuário, segundo os autores, haja maior dificuldade de acesso às informações, ao contrário do sistema informatizado, em que todo usuário registrado no sistema pode acessar a qualquer momento informações de pacientes, compartilhamos com eles o reconhecimento de que seja no suporte papel ou em prontuários eletrônicos, o fundamental é a qualidade das informações prestadas. Quando o registro no prontuário é escasso e inadequado a 
segurança do paciente, da instituição e da equipe ficam comprometidas e há dificuldade para mensurar os resultados assistenciais advindos das práticas profissionais envolvidas, como se verá a seguir.

Dos 29 prontuários analisados nesse estudo, na folha de Evolução Médica, apenas 22 continham a assinatura do médico responsável, enquanto que nos outros 7, a assinatura era do técnico de enfermagem ou não existia.

Somente 27 dos prontuários continham o Termo de Consentimento, contudo 23 destes não foram assinados pelo médico responsável.

Em relação à checagem das prescrições médicas, 19 prontuários foram assinados pelo médico responsável, enquanto 10 deles não possuíam qualquer assinatura ou tinham sido assinados pelo técnico de enfermagem.

Pautados em pesquisa em cinco hospitais em Recife/PE, Sampaio e Silva (2010), também encontraram que o preenchimento incorreto e/ou ilegível dos prontuários médicos tem sido um problema comum em grande parte dos hospitais brasileiros. Para os autores referidos, um prontuário preenchido com dados insuficientes

ou ilegíveis pode representar uma inadequada relação médico-paciente, que realizada de forma apressada e/ou superficial desrespeita os direitos do usuário. Determinantes dessa situação, devem ser buscados nas relações e nos processos de trabalho, na profissionalização inadequada, a não-adoção dos princípios éticos, bem como o desconhecimento e o respeito ao Sistema Único de Saúde (SUS) (SAMPAIO; SILVA, 2010).

Ainda que o trabalho de Sampaio e Silva (2010), acima citado, refira-se, exclusivamente, ao desempenho médico no preenchimento dos prontuários, os resultados do presente estudo, como se verá a seguir, indicam que os problemas não se restrigem apenas aos registros mantidos por esse profissional. Pelo contrário, as insuficiências são observadas também em relação aos outros membros da equipe multiprofissional e provavelmente, sejam determinadas pelas mesmas variáveis identificadas por Sampaio e Siva (2010).

O registro de Evolução Multidisciplinar foi realizado em 18 dos 29 prontuários e englobavam áreas: Enfermagem (2), Fisioterapia (4), Fonoaudiologia (2), Medicina (4), Nutrição (5), Psicologia (8) e Terapia Ocupacional (3), tendo sido assinadas pelos respectivos profissionais. As anotações desses vários profissionais revelaram que cada um deles informava sobre $\mathrm{o}$ atendimento aos pacientes, de maneira diversa $\mathrm{e}$ valorizando aspectos que permitiam identificar a origem da formação científica que 
receberam e utilizando o jargão da sua categoria profissional. Na maioria dos registros não havia indicação do solicitante ou do motivo da assistência (o quê, em alguns casos, podia ser deduzido a partir dos registros feito pelo profissional) e em vários não constava a data da anotação.

Em 7 prontuários a folha de Evolução Multidisciplinar estava em branco e, em outros 4, ela não constava.

Mesquita e Deslandes (2010), ao abordarem a construção dos prontuários como expressão da prática dos profissionais de saúde, apontam que práticas como as anteriormente citadas, sugerem que a maneira como alguns profissionais interpretam a função do prontuário, diverge da definição de prontuário como instrumento de registro de informações e de caráter obrigatório, como se não o compreendessem como documento de caráter também jurídico. A ausência de registros ou sua incompletude, conforme encontrado nos resultados da pesquisa aqui apresentada, prejudicam a transparência nos procedimentos empregados na assistência ao paciente e, portanto, não respaldam o valor ético e documental do prontuário.

Se esses aspectos já não fossem graves o bastante, lembramos também que, atualmente, é exigência do Programa Brasileiro de Acreditação Hospitalar a existência de prontuários completos, com informações de qualidade e disponíveis nas áreas assistenciais para certificação dos hospitais e no caso do hospital onde ocorreu o estudo isto é especialmente relevante, uma vez que o mesmo submete-se ao referido programa do Ministério da Saúde.

Os resultados específicos aos registros dos profissionais de psicologia mostraram que em 8 casos (prontuários) houve atendimento psicológico, sendo que em 3 deles a assistência foi prestada aos pacientes por solicitação da enfermagem e em 1, o alvo foi um membro da família do paciente (sem, no entanto, que qualquer anotação indicasse o motivo ou o solicitante).

Os registros referentes aos 4 pacientes são apresentados a seguir:

Prontuário1: A pedido da equipe de enfermagem, realizei contato com paciente a fim de coletar mais dados para planejamento de intervenção. Paciente consciente, orientado, comunicando-se por gestos. Apresentou demanda relativa à dificuldade de comunicação com equipe. Realizei acolhimento de demanda e informei que fico à disposição. Discuti caso com Dra. G. (fonoaudióloga) e solicitei avaliação do paciente. Mantenho acompanhamento. ( $1^{\mathrm{a}}$ anotação da Psicóloga 1$)$ 
Paciente consciente, orientado, animado e comunicativo. Relatou fatos da internação e da vida. Sem demanda emocional no momento. Informei que fico à disposição. Mantenho contato. ( $2^{\mathrm{a}}$ anotação da Psicóloga 1, dois dias depois).

Prontuário 2: Realizo acompanhamento com a paciente, com a presença da mãe, no momento da visita. Paciente apresenta-se agitada, ansiosa, chorosa, queixando-se de sede. Oriento sobre a necessidade da restrição hídrica neste momento do tratamento. A mãe da paciente apresenta episódio de choro intenso. Acolho e intervenho com objetivo de fortalecer a postura de enfrentamento da paciente e da sua mãe frente a hospitalização. Seguirão com acompanhamento. ( $1^{\mathrm{a}}$ anotação da Psicóloga 2).

Sou informada pela equipe da fisioterapia que a paciente está apresentando desconforto respiratório após a extibação (que ocorreu ás 15h) necessitando fazer inalação e não esforço para falar. Oriento a mãe da paciente, antes da visita, sobre as condições dela. Acompanho a visita, que ocorre sem interferência. Paciente e família seguirão com acompanhamento psicológico. ( $2^{\mathrm{a}}$ anotação da Psicóloga 2 , dez horas depois).

Realizo primeiro contato com paciente a fim de coletar mais dados sobre a mesma. Paciente orientada, consciente, comunicativa. relata fatos de sua história de vida, dinâmica familiar, motivo da internação e permanência intra-hospitalar. Apresenta ansiedade associada a longo período de internação e vivência em UTI. Realizo acolhimento de demandas presentes e intervenho com objetivo de auxiliar elaboração de enfrentamento de situação. Informo que fico a disposição. Mantenho contato. (Psicóloga 1, sem data).

Paciente consciente, orientada, comunicativa. Relata melhora em quadro de ansiedade após reintrodução de medicação. Sem demanda emocional no momento. Mantenho acompanhamento. (Psicóloga 1).

Paciente consciente, orientada, ansiosa, agitada, comunicativa. relata novos fatos de sua história de vida. Afirma utilizar sertralina e amitriptilina há cinco anos, porém, está sem a medicação desde que foi internada. Realizo acolhimento com paciente. Mantenho acompanhamento. Informo equipe de enfermagem e $\operatorname{Dr} G$ (urologista) quanto à medicação de uso contínuo referida pela paciente. (Psicóloga 1, sem data).

Paciente consciente, orientada, comunicativa. Relata recebimento de alta hospitalar, mostrando-se ansiosa pelo retorno ao lar. Realizo escuta ativa e incentivo enfrentamento de situação. Mediante alta, finalizo acompanhamento de 
presente internação. Informo que fico á disposição. (Psicóloga 1, sem data).

Prontuário 3: Paciente orientado e consciente. Refere estar tranquilo e arrumado (sic). Apresenta boa adesão ao tratamento. Buscou-se facilitar a expressão de sentimentos $e$ pensamentos. (Psicóloga 3)

Prontuário 4: Realizo avaliação com o sobrinho da paciente e, frente a postura adequada apresentada pelo mesmo, acompanho familiar em visita à paciente. Sem intercorrências. Ao final, acompanho familiar até a saída de visitantes, realizo acolhimento e informo que fico à disposição. (Psicóloga 1).

A análise desses registros mostra que ainda que as notas psicológicas sejam redigidas de modo claro, faltam informações sobre a subjetividade dos pacientes que não sejam relacionadas ao quadro clínico e tratamento, no momento da assistência prestada pelas psicólogas. Se como afirmam Almeida, Anderson e Costa Junior (2008), as informações colhidas pelos profissionais de Psicologia devem fazer parte do prontuário dos pacientes, de forma a adicionar uma dimensão mais funcional e humana, os registros analisados ao não permitirem a identificação da história do indivíduo na interface entre processo de adoecimento e situação psicossocial de forma compreensível, reforça a postura de especialista do profissional sem, contudo, colocar em questão o caráter comunicativo e coletivo dos registros.

Mais do que uma questão de necessidade de reafirmar sua identidade profissional ou preservar o sigilo de informações confidenciais, expondo apenas o necessário e o quê julga relevante no quadro do paciente ou como resposta a parecer(es) solicitado(s), acreditamos que os profissionais de psicologia, revelam em suas anotações, as condições e circunstâncias operacionais que lhe são apresentadas e às quais, por inúmeras razões, acabam por se submeter.

Nesse sentido, constatamos, como em estudo anterior (Prebianchi, 2015) que a inserção dos psicólogos na equipe interdisciplinar, ocorre mediante solicitações de interconsulta para casos específicos nos quais exista dificuldade de adesão ao tratamento médico e/ou quando os pacientes ou seus familiares apresentam comportamentos com os quais a equipe não sabe lidar e que são disfuncionais ao cotidiano das práticas de cuidados e do funcionamento das enfermarias. Além disso, o fato de diferentes profissionais psicólogos atuarem com os pacientes (e às vezes com um mesmo paciente), revela que, de fato, a atenção integral aos pacientes cirúrgicos fica 
reduzida a ações distintas realizadas por especialistas de diversas áreas, em diferentes momentos e de modo fragmentado, disvirtuando a interdisciplinaridade.

Nossa asserção anterior encontra respaldo também quando consideramos a ausência de registros nos outros 4 prontuários de pacientes que foram atendidos pelos psicólogos. A partir do nosso estranhamento causado pela ausência de anotações nos prontuários, descobrimos, junto à chefe do Serviço de Psicologia, havia um caderno de registro próprio da área (Livro da Psicologia) ao qual somente os psicólogos têm acesso. Ao examinarmos o referido caderno, descobrimos que nele constavam as anotações que faltavam nos 4 prontuários. Esse aspecto é similar ao encontrado por Mesquita e Deslandes (2010) em seu estudo, no qual apontam que a ausência de anotações dos diversos componentes da equipe de saúde nos prontuários, devia-se ao fato que cada categoria profissional mantinha anotações em cadernos de registro e agendas, próprios de cada área e mantidos em sigilo nas respectivas salas desses profissionais. As autoras concluíram que a equipe não se comunicava através de prontuários, fruto de uma prática que, particularmente, não contribuía para o desenvolvimento de ações interdisciplinares, o quê julgamos também se aplique aos resultados aqui apresentados.

\section{Conclusão}

Partindo do reconhecimento da importância de se desenvolver, estabelecer e aperfeiçoar modos sistematizados de atendimento à saúde do paciente cirúrgico, que incluam a estruturação de mecanismos que viabilizem e melhorem o sistema de comunicação interdisciplinar, nosso objetivo foi investigar a comunicação entre os membros da equipe interdisciplinar na assistência aos pacientes adultos submetidos a cirurgias eletivas, através dos registros nos prontuários desses pacientes.

Ao final, concluímos que a baixa qualidade dos prontuários em termos de sua completude e da adequação de suas anotações, não inviabilizou nosso trabalho, mas sim, nos revelou quão distante, a maior parte das instituições de saúde, especificamente, os hospitais, se encontra da efetivação das práticas interdisciplinares preconizadas pelas políticas públicas e pelo modelo biopsicossocial de atenção à saúde. Ainda que nosso estudo tenha se desenrolado num único hospital, permitimo-nos certo grau de generalização, baseados na literatura relativa ao tema na realidade nacional. 
A qualidade insatisfatória dos documentos analisados (nesse e em outros estudos já citados), impede que sirvam de meio de comunicação compartilhado tanto entre todos os profissionais de saúde, aproximando a equipe de um contexto de trabalho interdisciplinar, quanto da equipe com o paciente (lembrando que a ele pertence o prontuário).

E se considerarmos as anotações ou ausência delas como reflexo das práticas dos profissionais reproduzidas a partir de sua formação e, das condições e circunstâncias operacionais que lhe são apresentadas, podemos concluir que no hospital, contexto de nossa pesquisa, os profissionais da equipe mantêm o caráter de especialistas, com forte delimitação do seu papel individual graças à distorção da lógica da interdisciplinaridade. Nesse caso, a atenção ao paciente cirúrgico é realizada por uma equipe cujo trabalho se caracteriza pela centralidade da figura do médico e numa visão fragmentada e binária dos vários especialistas - o quê reforça o modelo médico-biológico de atenção à saúde.

Finalmente, o verdadeiro descaso dos profissionais envolvidos para com o adequado preenchimento dos prontuários, diverge da definição do documento como instrumento de registro de informações, de caráter obrigatório, que funciona como mediador da comunicação entre profissionais e usuários, preservando a relação ética entre os sujeitos envolvidos.

Para além da questão ética, acreditamos que se a comunicação escrita no prontuário documenta, de modo permanente, as informações relevantes sobre os cuidados prestados ao paciente, negligenciá-la é desconsiderar o caráter jurídico do instrumento e desprezar a avaliação da qualidade e da eficácia da assistência prestada. Assim, propomos seja dado treinamento aos profissionais da equipe, pela instituição, a fim de esclarecê-los e conscientizá-los sobre o tema, bem como para promover a utilização de escrita em formato que proporcione uma comunicação interdisciplinar efetiva.

Todavia, temos clareza de que sem o desenvolvimento e o aperfeiçoamento do sistema de saúde do Brasil, proporcionado por maiores investimentos no setor e melhoria nas formas e mecanismos de gestão hospitalar, mudanças substantivas no cuidado e elaboração dos prontuários, certamente não garantirão a assistência integral aos pacientes. Por outro lado, a boa qualidade dos registros efetuados poderá salvaguardar os aspectos éticos e jurídicos das relações de cuidados e impedir a continuidade dos reflexos negativos sobre as áreas de ensino e pesquisa. 


\section{REFERÊNCIAS}

ALMEIDA, F. F.; ADERSON, C. C.; COSTA JUNIOR, L. Prontuário psicológico orientado para o problema: um modelo em construção. Psicologia: ciência e profissão, 28 (2), 430-442, 2008.

AZEVEDO, F. M.; THOMAS, C. V. Registro psicológico em prontuário. Revista da Sociedade Brasileira de Psicologia Hospitalar, 5 (1/2), 26-28, 2002.

BROERING, C. V.; CREPALDI, M. A. Preparação psicológica e o estresse de crianças submetidas a cirurgia. Psicologia em Estudo, Maringá, 16 (1), 2011.

CALVETTI, P. U.; FIGHERA, J.; MULLER, M. C. A bioética nas intervenções em psicologia da saúde. PSIC. Revista de Psicologia da Vetor Editora, 9 (1), 115-120, 2008.

CONSELHO FEDERAL DE MEDICINA. Resolução no.1638/2002, de 10 de julho de 2002. Define prontuário médico, responsabilidade médica e criação de Comissão de Revisão de Prontuário Médico. Disponível em: <http://www.protalmedico.org.br/resolucoes/dfm/2002/1638_2002.htm>. Acesso em: 29 mar. 2015.

DALL'AGLIO, J. S.; MARTINS, Z. Avaliação e dados de Prontuários do Módulo Urgência e Emergência do Hospital de Clínicas da Universidade Federal de Uberlândia. Revista da Católica, 3 (5), 1-16, 2011.

DESLANDES, S. F. Frágeis Deuses: profissionais da emergência entre os danos da violência e a recriação da vida. Rio de Janeiro: Editora Fiocruz, 2002.

FERRACINI, A.; PREBIANCHI, H. B. (no prelo). Psicoprofilaxia cirúrgica de pacientes infantis e adolescentes: percepção dos médicos. Psicologia em Revista.

GAZOTTI, T. C.; PREBIANCHI, H. B. Caracterização da interconsulta psicológica em um hospital geral. Psicologia: Teoria e Prática, 16 (1), 18-30, 2014.

GIOIA-MARTINS, D. F.; ROCHA, A. J. Psicologia da saúde e o novo paradigma: novo paradigma? Trabalho apresentado no I congresso de Psicologia clínica, São Paulo, Brasil.

JUAN, K. Psicoprofilaxia cirúrgica em urologia. Psicologia Hospitalar (São Paulo), 3 (2), 1-10, 2005.

KLÜCK, M. M.; GUIMARÃES, J. R. Prontuário de pacientes: finalidades, preenchimento e questões éticas e legais. In: BARROS, E.; ALBUQUERQUE, G.; PINHEIRO, C.; CZEPIELEWSKI, M. A. Exame clínico. Consulta rápida. Porto Alegre: Artmed, 2014.

MESQUITA, A. M. O.; DESLANDES, S. F. A construção dos prontuários como expressão da prática dos profissionais de saúde. Saúde e sociedade, 19 (3), 664-673, 2010. 
NASCIMENTO, A. L.; ANDRADE, M. G.; ASSED, A. M. P.; BRASIL, M. A. A. Um modelo para o parecer psiquiátrico no hospital geral. Jornal Brasileiro de Psiquiatria, 55(2), 102-107, 2006.

SAMPAIO, A. C.; SILVA, M. R. Prontuários médicos: reflexo das relações médicopaciente. Revista Bioética, 18(2), 451-468, 2006.

SILVA, F. G.; TAVARES-NETO, J. Avaliação dos prontuários médicos de hospitais de ensino do Brasil. Revista Brasileira de Educação Médica, 31 (2), 113-126, 2007.

TURRA, V.; COSTA JUNIOR, A. L.; ALMEIDA, F. F.; DOCA, F. N. P. Contribuições da Psicologia na atenção ao paciente cirúrgico: uma análise da literatura. Com.

Ciências Saúde, 22(4), 353-366, 2011.

\section{Como referenciar este artigo}

PREBIANCHI, Helena Bazanelli. Comunicação da equipe interdisciplinar na assistência aos pacientes cirúrgicos. Temas em Educ. e Saúde, Araraquara, v.13, n.1, p. 164-178, jan./jun. 2017. DOI <https://doi.org/10.26673/rtes.v13.n1.janjun2017.11.9471>. ISSN: 1517-7947.

Submetido em: 28/02/2017

Aprovado em: 20/06/2017 